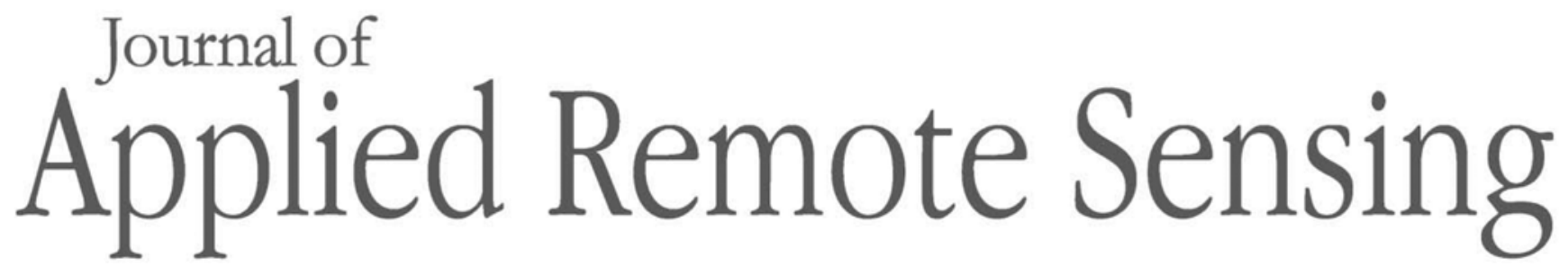

RemoteSensing.SPIEDigitalLibrary.org

\title{
Integrating seasonal optical and thermal infrared spectra to characterize urban impervious surfaces with extreme spectral complexity: a Shanghai case study
}

\author{
Wei Wang \\ Xinfeng Yao \\ Minhe Ji
}

\section{SPIE.}




\title{
Integrating seasonal optical and thermal infrared spectra to characterize urban impervious surfaces with extreme spectral complexity: a Shanghai case study
}

\author{
Wei Wang, ${ }^{a, \dagger}$ Xinfeng Yao, ${ }^{b, \dagger}$ and Minhe $\mathbf{J i}^{\mathrm{a}, *}$ \\ ${ }^{a}$ East China Normal University, Key Lab of GIScience, Education Ministry of China, \\ 500 Dongchuan Road, Minhang District, Shanghai 200241, China \\ ${ }^{b}$ Agricultural Information Institute of Science and Technology, Shanghai Academy of \\ Agricultural Sciences, 1000 Jinqi Road, Fengxian District, Shanghai 201403, China
}

\begin{abstract}
Despite recent rapid advancement in remote sensing technology, accurate mapping of the urban landscape in China still faces a great challenge due to unusually high spectral complexity in many big cities. Much of this complication comes from severe spectral confusion of impervious surfaces with polluted water bodies and bright bare soils. This paper proposes a twostep land cover decomposition method, which combines optical and thermal spectra from different seasons to cope with the issue of urban spectral complexity. First, a linear spectral mixture analysis was employed to generate fraction images for three preliminary endmembers (high albedo, low albedo, and vegetation). Seasonal change analysis on land surface temperature induced from thermal infrared spectra and coarse component fractions obtained from the first step was then used to reduce the confusion between impervious surfaces and nonimpervious materials. This method was tested with two-date Landsat multispectral data in Shanghai, one of China's megacities. The results showed that the method was capable of consistently estimating impervious surfaces in highly complex urban environments with an accuracy of $R^{2}$ greater than 0.70 and both root mean square error and mean average error less than 0.20 for all test sites. This strategy seemed very promising for landscape mapping of complex urban areas. () The Authors. Published by SPIE under a Creative Commons Attribution 3.0 Unported License. Distribution or reproduction of this work in whole or in part requires full attribution of the original publication, including its DOI. [DOI: 10.1117/1.JRS.10.016018]
\end{abstract}

Keywords: impervious surfaces; land cover decomposition method; seasonal change analysis; land surface temperature.

Paper 15779 received Nov. 11, 2015; accepted for publication Feb. 2, 2016; published online Feb. 25, 2016.

\section{Introduction}

Modern cities are necessarily diverse and complex in their physical compositions and morphological structures to support urban dwellers' well-beings and development. In order to minimize analytical uncertainty, many remote sensing studies on urban impervious surface extraction tend to choose cities with simple urban structures and low fragmentations as their research objects in Western countries. ${ }^{1-3}$ This strategy may not work well in Chinese cities with long histories, which are often characterized by an unusually complex morphology of high-density buildings, intermixed ground materials, and fragmented land use patterns; for instance, the city of Shanghai. Due to the current accelerated urbanization in China, the dynamics of impervious surfaces require accurate determination in a short time interval for comprehensive urban planning and environmental management, which poses a great technical challenge to remote sensing practitioners. ${ }^{4,5}$

A direct consequence of extreme urban heterogeneity is some serious interclass spectral confusion. ${ }^{1}$ Among all possible confusion that may affect the quality of complex urban

\footnotetext{
*Address all correspondence to: Minhe Ji, E-mail: mhji@geo.ecnu.edu.cn

${ }^{\dagger}$ These authors contributed equally to this work.
} 
landscape characterization, two major issues raise our concerns. The first issue is related to the identification and removal of water bodies from being involved in urban land cover characterization, which is a common practice when Ridd's vegetation-impervious surface-soil (V-I-S) model is adopted. ${ }^{3,6}$ This preprocessing task is, however, extremely difficult to conduct for urban areas in China, especially when numerous water bodies with drastically diverse spectral patterns in an image scene need to be processed. Failure in water removal may lead to unexpected errors in the subsequent analysis. ${ }^{7}$

The most commonly used strategies for extracting water bodies from remotely sensed images are based on the threshold of a single spectral band ${ }^{8}$ or spectral indices (e.g., normalized different water index and modified normalized different water index). ${ }^{9,10-12}$ However, it is very challenging to accurately define optimal thresholds for water extraction, because thresholds are scenebased and mostly depend on the fractions of subpixel water/nonwater components. ${ }^{8}$ Water bodies have spectral properties very similar to low-albedo (LA) impervious surface materials and shadows. ${ }^{13}$ Therefore, relying solely on optical spectra is limited and insufficient for complete separation of water bodies from other land cover features.

Recent studies have noted that the use of thermal infrared (TIR) data may be able to assist in separating water bodies from impervious surfaces, because the latter can emit more heat than the former, yielding an identifiable temperature difference between them. Lu and Weng ${ }^{13}$ scatterplotted land surface temperature (LST) against LA fraction images to separate water bodies from LA impervious surfaces. In other studies, the TIR band was engaged in building new spectral indices to enhance impervious surfaces by suppressing other land cover noises. ${ }^{7,14}$ Some more recent studies further revealed that thermal properties could be detected at the subpixel level, as LST variations can be closely related to land cover fractions. ${ }^{15-17}$ In short, LA impervious surfaces and water bodies may be separated by considering their differential contributions to LST.

The second issue in characterizing impervious surfaces is related to soil, one of the three urban land cover components in Ridd's model. ${ }^{18}$ Previous studies pointed out that bare soil has spectral response patterns highly similar to high-albedo (HA) impervious surfaces. ${ }^{3,7}$ Spatial stratification by the urban/rural dichotomy was used to distinguish between HA imperviousness and bare soil by the location of the pixel under examination. ${ }^{19}$ This approach has, however, proven to be much less effective in China's cities under continual development, as it is difficult to demarcate a definite boundary between urban and rural areas. Some other attempts to separate urban built-ups from bare land include enhanced built-up and bareness index ${ }^{14}$ and normalized difference bareness index. ${ }^{17}$ Due to high spectral variability among different types of impervious surfaces, however, these spectral indices have failed to provide satisfactory discrimination between impervious surfaces and bare soils in cities with extreme structural and spectral complexity.

Soil identification, on the other hand, may be better achieved with multitemporal imagery, which has been widely used for distinguishing land cover types with similar spectral characteristics on a single image. ${ }^{2,20}$ Plant phenology is hereby used as a primary basis for the soil-imperviousness distinction. Specifically, bare soils typically mix with leaf-off deciduous vegetation in a spring image, whereas the latter reaches a full coverage in a summer image. The difference between these two seasonal states is then derived to separate impervious surfaces from bare soils that have similar spectral characteristics in springtime. ${ }^{2}$ Due to the widespread existence of mixed pixels in a highly complex urban landscape ${ }^{21}$ the effectiveness of this pixel-based technique suffers a great deal when applied in a city like Shanghai. Instead, change detection in the form of fractions from using subpixel classification techniques may help to cope with the mixedpixel problem for better separation of impervious surfaces from bare soil.

In this study, a two-step land cover decomposition method is designed to combine seasonal optical and TIR data for impervious surface mapping in highly complex urban landscapes. We hereby use Shanghai as a typical case of extreme confusion among water, soil, and impervious surfaces to demonstrate the effectiveness of this new approach. In the main body of this text, two key issues will be addressed in detail: (1) how to remove water bodies from the LA fraction image, and (2) how to remove bare soils from the HA fraction image. This will be followed by a comparative case study to demonstrate the advantage of this method over the traditional ones. 
Wang, Yao, and Ji: Integrating seasonal optical and thermal infrared spectra to characterize urban...

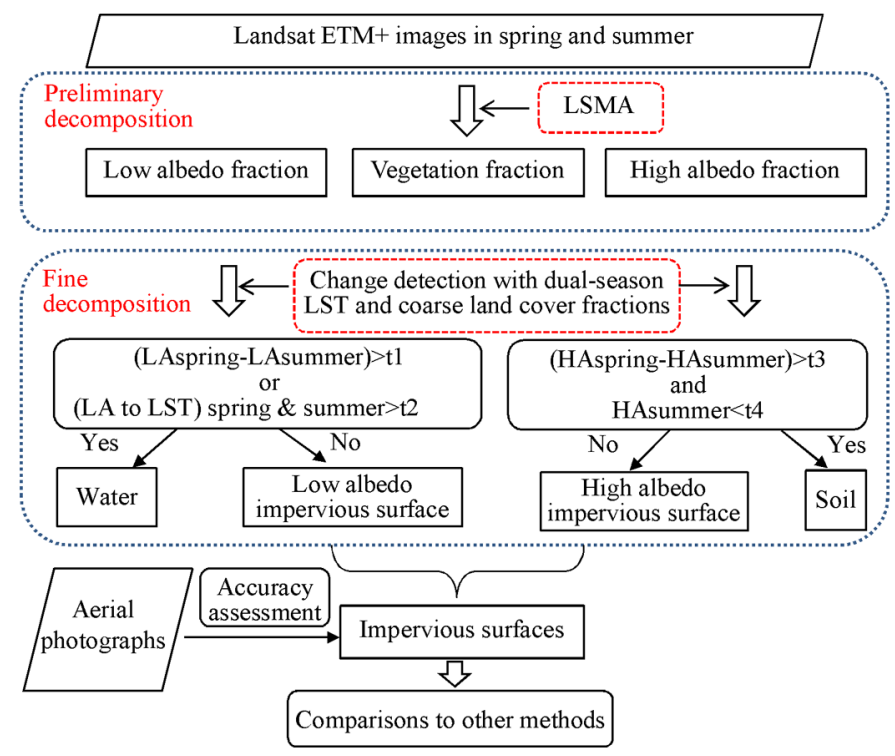

Fig. 1 Framework of the two-step land cover decomposition method.

\section{Methodology}

The proposed land cover decomposition method is based on a two-step classification approach. The first step extracts coarse land cover components (HA, LA, and vegetation) from optical multispectral data of both spring and summer at the subpixel level with the most commonly used linear spectral mixture analysis (LSMA). These coarse components contain a mixture of targeted fine-level materials that are spectrally inseparable in the first round of classification. In the second step, we integrated two-season coarse components (HA, LA, and vegetation) extracted in the first step and LST retrieved from TIR data to remove water bodies from LA as well as soils from HA for a better classification of impervious surfaces (Fig. 1). As a result of this postdecomposition processing, the LA impervious surface fraction now ideally contains only dark impervious surfaces, and the HA impervious surface fraction represents only bright impervious surfaces. Details of the data processing methods in the decomposition scheme are provided in Secs. 2.1, 2.2, and 2.3.

\subsection{Derivation of Preliminary Land Cover Fractions}

The proposed design requires preliminary land cover fractions of two chosen dates. These products are obtained by applying LSMA to each of the two selected optical multispectral datasets. The LSMA model is formally expressed as

$$
R_{m}=\sum_{i=1}^{n} f_{i} R_{i m}+\varepsilon_{m},
$$

where $i=1,2, \ldots, n, n$ is the number of endmembers; for each pixel, $R_{m}$ is the reflectance for band $m ; R_{i m}$ is the spectral reflectance of band $m$ for endmember $i ; \varepsilon_{m}$ is the error for band $m ; f_{i}$ is the fraction of endmember $i$; for a constrained unmixing solution, $f_{i}$ is subject to the following restrictions:

$$
\begin{gathered}
\sum_{i=1}^{n} f_{i}=1, \\
f_{i} \geq 0 .
\end{gathered}
$$

Due to the spectral confusion between water and LA imperviousness and between soil and HA imperviousness, the decomposition process at this step is intended to target only three coarse 
Wang, Yao, and Ji: Integrating seasonal optical and thermal infrared spectra to characterize urban...

endmembers of the scene, i.e., HA, LA, and vegetation. In this way, identifying pure pixels for these three coarse components is much easier than the traditional attempt to directly split Ridd's V-I-S components. Equation (1) assumes that the reflectance of any pixel is a linear combination of the reflectance of each endmember, i.e., HA, LA, and vegetation. The derivation process follows the usual procedure. First, a minimum noise fraction (MNF) transform of the original optical multispectral data is performed, and the first two MNF are mapped into a 2-D scatterplot for visual identification of spectral features related to the three coarse components. Second, pixels comprising these three spectral features in the MNF space are extracted as endmembers to build an LSMA model, which is in turn used to generate three fraction images, with each representing the abundance of HA, LA, and vegetation, respectively.

\subsection{Derivation of Fine Land Cover Fractions}

Two of the three preliminary land cover fractions resulting from the above step contain a mixture of targeted fine land cover fractions. Specifically, the LA consists of water and LA impervious surface, and the HA is composed of soil and HA impervious surface. To deal with these component mixtures, a postdecomposition classification was constructed with two-season LST and coarse fraction images to detect water bodies from LA and soils from HA.

\subsubsection{Identifying perennial water by comparing low albedo fraction-to-land surface temperature ratio}

The mixture of water and LA imperviousness is inevitable due to their close values in the optical bands; however, the former is usually much lower in LST than the latter, since their thermal behaviors can be drastically different. This LST difference can therefore be used to stretch the contrast between water and LA imperviousness by a ratio of LA fraction to LST. It is reasonable to assume that, for any specific pixel, the LA fraction-to-LST ratio will be low with a high percent of coverage of LA impervious surface and high for LA composed of water. The greater the ratio value, the higher the possibility of a pixel being mixed with water. In this study, LA fraction and LST were adopted to produce a ratio map for each season. An identical threshold value was determined for both the spring and summer ratio maps to filter out perennial water bodies (e.g., rivers and ponds) from the LA fraction map. Comparing the two ratio maps may also weed out shadows, as shadows may not maintain high ratio values due to variations in shadow length and orientation from season to season. To account for the uncertainty involved in derivation of component fractions for two different seasons, a scheme was developed to determine a proper LA fraction-to-LST ratio threshold. That is, the aerial photographs were utilized as the major reference data to derive the fine land cover information, which was used to compare with the corresponding LA fraction-to-LST ratio map, and a manual trial-and-error approach was applied to select the proper thresholds for perennial water extraction in this study.

\subsubsection{Identifying seasonal water by comparing seasonal low albedo fraction maps}

The threshold of LA fraction-to-LST ratio for filtering perennial water bodies in spring and summer images may not work effectively for seasonal water bodies in suburban areas, such as seasonally-submerged rice paddies and wetlands alternately covered by water and vegetation throughout the year. Therefore, this study designed a change detection procedure that uses both spring and summer seasonal LA fraction images to extract seasonal water features. It is based on the rationale that the spring-to-summer transition of LA fraction might be able to signify the difference between seasonal water bodies, such as flooded rice paddies and fish ponds, and LA impervious surfaces. In early summer (June in the Yangtze River Delta), rice paddies are filled with water (more than $5 \mathrm{~cm}$ deep) for seedling transplanting, making them spectrally similar to shallow water. This situation usually continues through the first one-third of the growing season of rice, including tilling, and jointing, which can last for two months. 
Wang, Yao, and Ji: Integrating seasonal optical and thermal infrared spectra to characterize urban...

In comparison, the dominating crops in spring are winter wheat and rapeseed, both being planted in dry fields and less likely to appear as water. In addition, some temporary manmade ponds for fish farming in summer are usually dried up in spring, resulting in seasonal water bodies on the images. In this work, an area was deemed seasonal water if the LA fraction increases significantly from spring to summer, and this was determined through a carefully selected threshold value for the seasonal LA fraction difference. Similar to the threshold determination for the LA fraction-to-LST ratio, a manual trial-and-error procedure was followed to achieve this threshold.

\subsubsection{Identifying soil by comparing seasonal high albedo fraction maps}

Soil shares similar spectral characteristics with HA imperviousness, making them inseparable during the usual LSMA in our study area. However, their separation can be achieved by comparing coarse land cover fraction maps across multiple seasons. The temporal trajectory of a pixel across multitemporal land cover fraction images can provide important clues that a single-date image may not exhibit. Specifically, in this study, vegetation phenology plays a key role in revealing the identity of soil via HA change detected from a spring image and a summer image. In spring, most of the soils located in urban-rural fringes were bare and dry, showing up as part of the HA land cover. These pixels, however, changed into the vegetation cover type in summer due to the blooming herbs in the field, leading to low HA fraction values in the summer image. In this case, therefore, we assumed the pixels with spring-high and summer-low HA fraction values to be soil pixels and verified this assumption through in situ data. Soil identification was thus achieved by a filter consisting of a low summer HA fraction and a large seasonal HA fraction difference. Conversely, pixels with steady HA fraction values in both spring and summer would be considered to truly contain imperviousness. The in situ data was composed of a sample set of aerial photographs to obtain training pixels for establishing the change patterns of the HA fraction from spring to summer. This training process resulted in a pair of proper threshold values to separate the true HA impervious surface from soil.

\subsubsection{Generation of impervious surface fraction map}

The impervious surface fraction for each of the two seasons was generated by simply summing the preprocessed LA and HA impervious surface fractions of that season, with water and soil being removed in the previous steps.

\subsection{Accuracy Assessment}

In order to accommodate the spatial variability of the urban landscape in Shanghai, accuracy assessment was performed on two selected areas, representing urban and rural types, respectively. We stratified the samples by the areal proportion of each land cover class within the study area. The impervious estimates resulting from the analysis of the ETM+ images were compared to their much finer counterparts visually interpreted from the high-resolution digital images of the same or adjacent year. To avoid geometric errors, the resolution of the aerial photography was downscaled from 0.6 to $9.0 \mathrm{~m}$ for the convenience of visual comparison. The size of each sample was set to $10 \times 10$ pixels, which equaled $90 \times 90 \mathrm{~m}$, i.e., $3 \times 3 \mathrm{ETM}+$ pixels. A total of 100 sites for each typical area (i.e., urban and rural) were sampled randomly. The reference fraction is derived from the aerial photographs as follows. First, for each sample window with size of $10 \times 10$ pixels, the impervious pixels were visually determined. Second, the total number of impervious pixels in each sample window was calculated. Third, the impervious surface fraction was computed by dividing the number of impervious pixels by the total number of pixels within the sample window. Regression analyses and scatter plots were applied to evaluate the fitness between the estimated impervious surfaces and reference data. Three indices were utilized to evaluate the accuracy for the impervious surfaces estimation: the root mean square error (RMSE), mean average error (MAE), and correlation coefficient of determination $\left(R^{2}\right)$, using the following equations, respectively 


$$
\begin{gathered}
\text { RMSE }=\sqrt{\frac{\sum_{i=1}^{n}\left(P_{i}-O_{i}\right)^{2}}{n},} \\
\text { MAE }=\frac{1}{n} \sum_{i=1}^{n}\left|P_{i}-O_{i}\right|, \\
R^{2}=\frac{\left(\sum_{i=1}^{n}\left(P_{i}-\bar{P}\right)\left(O_{i}-\bar{O}\right)\right)^{2}}{\sum_{i=1}^{n}\left(P_{i}-\bar{P}\right) \sum_{i=1}^{n}\left(O_{i}-\bar{O}\right)},
\end{gathered}
$$

where $P_{i}$ is the estimated impervious fraction value for sample $i, \bar{P}$ is the mean value of all estimated samples, $O_{i}$ is the reference value obtained from the aerial photographs for sample $i, \bar{O}$ is the mean value of all reference samples; and $n$ is the total number of samples.

\section{A Case Study of Shanghai}

\subsection{Study Sites and Data}

The aforementioned approach was tested in Shanghai, a megalopolis situated on the east coast of China and geospatially ranging from $31^{\circ} 32^{\prime} \mathrm{N}-31^{\circ} 27^{\prime} \mathrm{N}$ to $120^{\circ} 52^{\prime} \mathrm{E}-121^{\circ} 45^{\prime} \mathrm{E}$, with a land area of more than $6340 \mathrm{~km}^{2}$ and a population of over 19 million in 2012. While the well-built central area is confined inside the outer loop highway, the current urban boundaries have extended far beyond the outer loop, with the recent major urban sprawl taking place outside the loop. ${ }^{22,23}$ Shanghai can be viewed as being composed of three different developmental patterns, including a highly developed commercial and residential center (circumscribed by the outer loop) with high-density population and buildings; suburban areas surrounding the city center, now being converted to "new cities"; and rural areas located outside urban cores of various scales and mainly characterized by crop fields (Fig. 2). After evolving through several different regimes over more than a century, the city has now presented a typical amalgamation of old and new land uses, ground materials, and urban structures. There is a heavy mixture of diverse land use types in the urban and suburban areas, such as parks, settlements, croplands, grasslands, and forests. Most buildings in the urban core are old and fragmented, whereas those in the newly developed regions are more regularly shaped and oriented. The braided river system is intricate and highly

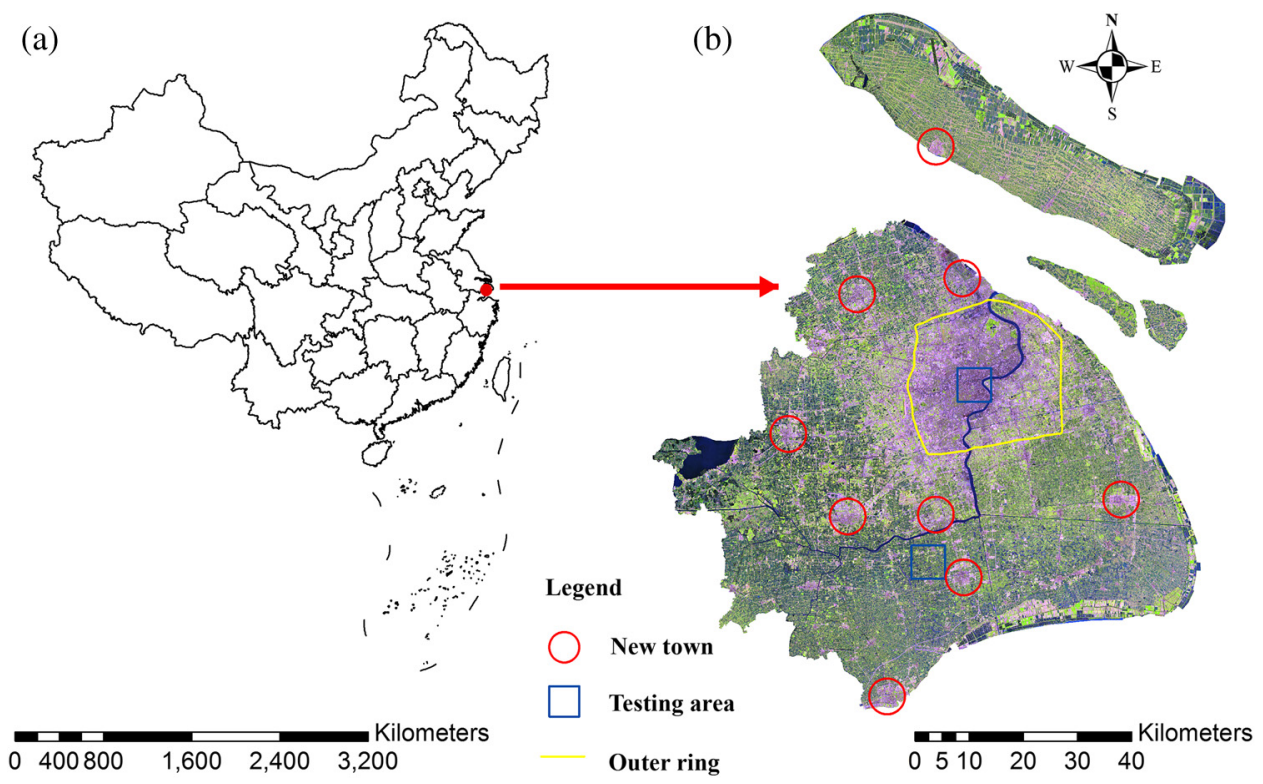

Fig. 2 Study area: (a) location in China and (b) geographical extent as illustrated with an RGB-543 color composite of Landsat ETM+ image. 
Wang, Yao, and Ji: Integrating seasonal optical and thermal infrared spectra to characterize urban...

convoluted at the tip of the Yangtze Delta. After decades of land use change and urbanization, this braided river system has been seriously severed into numerous isolated stream segments and small ponds in the city. With variations in size, water depth, and pollution level, these water bodies present a severe spectral complication. On the other hand, the agricultural features vary with soil conditions and plant phenology. Rice is the dominant crop type in summer and autumn, mixed with shallow water in the field for a long time during the growing season, and rapeseed and winter wheat are the principal crops in winter and spring.

The primary data consisted of a temporal pair of cloud-free Landsat ETM+ images (path 118/row 38, path 118/row 39) acquired on 13 March 2001 and 3 July 2001. The data were obtained from the USGS Earth Resource Observation Systems Data Center ${ }^{24}$ and rectified to a Universal Transverse Mercator (UTM) coordinate system with the WGS84 datum and UTM zone 51. The Landsat ETM+ optical data (bands 1 to 5 and 7) and thermal data (band 6) are utilized in this study. Note that spatial resolution for the thermal infrared (ETM + band 6) during image acquisition is 60 meters, but this band was resampled to a 30-m pixel size in this study to be consistent with the spatial resolution of optical bands. A visual inspection of the overlaid optical and thermal bands indicated that they achieved a good geometric matching precision with a registration error of less than one pixel. The Google Earth high-resolution images of 2001 were used for visual reference, and a set of 0.6-m color-infrared aerial photographs, dated March and April of 2000, were used for the quantitative validation purposes.

\subsection{Image Preprocessing}

The digital numbers of both optical and thermal data were first converted to at-satellite radiance according to the Landsat science data user's handbook, then were converted to spectral reflectance by the FLAASH atmospheric correction model. The thermal data was converted to LST by employing the single-channel algorithm. ${ }^{25}$

\section{Results}

\subsection{Spatio-Temporal Patterns of Land Surface Temperature}

The LST distribution patterns for both spring and summer of 2001 in Shanghai are shown in Fig. 3, where the same symbolization was adopted to represent different value ranges due to the fact that the spring and summer temperatures do not overlap (i.e., 285.6-290.8 $\mathrm{k}$ for spring and 293.6-300.9 k for summer). Despite the drastic difference in value ranges, the spatial patterns of temperature distribution appear highly similar. In both spring and summer, the highest LST values (displayed in red) are mostly clustered in the central business district (CBD) area. The apparent hot spots in the suburban areas also signify the widespread development of new cities. The exurban areas show a sporadic distribution of small hotspots, corresponding to townships and large rural settlements where urbanization is minimal. It is observed that the road networks connecting central urban areas and new cities exhibit an LST higher than their surrounding vegetated areas. The low-LST pixels (displayed in blue) are found in nondeveloped areas, in which water bodies, forest, and agriculture are dominant land covers.

In order to examine the LST variations in detail, two test sites, one in the CBD area and the other in the rural area (shown in Fig. 2 within the blue rectangle), were selected for comparative analysis [Fig. 3(c)]. Visual evidence provided further observations when the two seasons were compared. For the urban site, the seasonal differences in LST were mostly induced by the seasonal change of vegetation and shadow. It can be seen from the spring map that low temperatures occurred in the vegetation, water, and shadow areas, but in the summer, the cooler pixels increased with vegetation growth, and obviously decreased in the previous shadow pixels due to shorter shadows in summer. For the rural site, the spring map displays a much higher LST variability in vegetated areas than the summer map. This seems attributable to the fact that spring farmlands were dominated by winter crops (i.e., wheat and rapeseed) mixed with dry and bare soil in the background, resulting in a relatively high and uneven emissivity in the thermal 


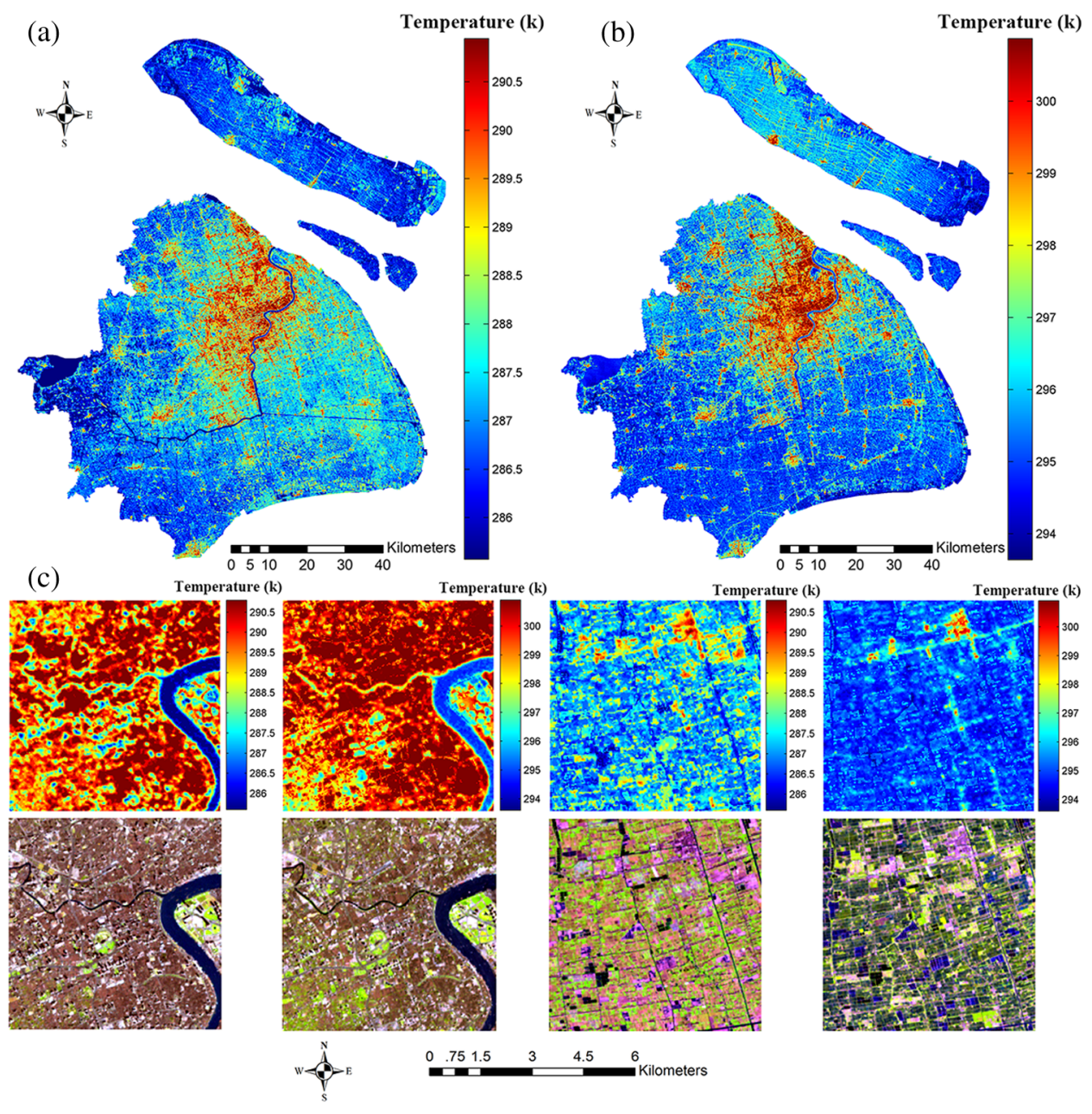

Fig. 3 Spatial patterns of LST in Shanghai on (a) March 13, 2001, and (b) July 3, 2001. The enlarged LST maps in the first row in (c) from left to right are urban area in spring, urban area in summer, rural area in spring, and rural area in summer, respectively, and their corresponding original ETM+ images with a color composite RGB $=543$ in the second row.

band. In summer, however, most of the farmlands in the region were shifted to flooded rice paddies, which possessed a lower and more evenly distributed LST, owing to shallow water in the field.

\subsection{Preliminary Extraction of Urban Compositions}

The preliminary extraction of urban compositions was made by applying a three-endmember LSMA. Endmembers were identified from the vertices of the MNF-based scatterplots, and the number of selected pure pixels of HA, LA, and vegetation, respectively, is: 918, 1125, and 1029 in spring and 877, 933, and 1392 in summer. Then, endmember fractions of HA, LA, and vegetation were estimated for the two seasons following the procedure described in Sec. 2.1. The seasonal difference maps of component fractions are provided in Fig. 4. HA are associated with objects that have very high reflectance values, such as bright roofs, construction materials, bare soils, and newly built areas. The green color in Fig. 4(a) indicates little to no HA fraction changes in those areas. But there is also a significant reduction of HA fraction in agricultural lands that are bare soil or mixed with soil in spring and are changed to rice paddies in summer [displayed in blue in Fig. 4(a)].

The LA mainly corresponded to the objects with very low reflectance. This component contained multiple land cover types, including water bodies and shadows of vegetation canopies and tall buildings, in addition to LA imperviousness. The performance of the perennial water bodies 

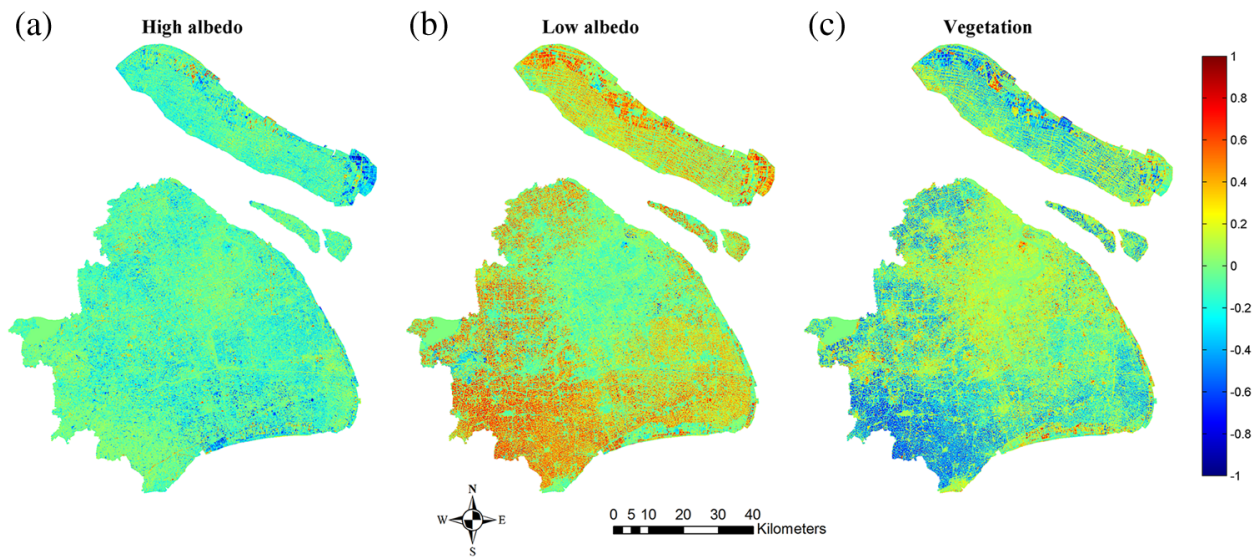

Fig. 4 Fraction difference maps from March 13, 2001, to July 3, 2001: (a) HA, (b) LA, and (c) vegetation.

was time-insensitive (highlighted in green), such as the Huangpu River, winding through the heart of Shanghai, and Dianshan Lake, located in the western suburb of Shanghai. LA fraction in the CBD and other major business centers, as well as old residential neighborhoods, demonstrated insignificant seasonal variation as well [Fig. 4(b)]. The only subtle change in these areas is the small but overall spring-to-summer decrease of fraction values due to the growth of trees in July. The major seasonal difference in this category is evident in the rural areas, where a general spring-to-summer increase in LA fraction was observed. This is because of the crop rotation in the field: water-dependent rice in summer replacing the dry-climate winter wheat and rapeseed in spring. Shallow water in the rice paddies produces high LA fraction values, making these pixels stand out in the seasonal difference map [Fig. 4(b)].

Visually inspecting the seasonal difference fraction map of vegetation revealed that the fractions changed more drastically in the rural areas than in the urban areas [Fig. 4(c)]. The minor fraction increase in the urban core from spring to summer resulted from the vegetation phenology, whereas the drastic decrease in the exurbs is more related to crop rotation. The summer vegetation coverage in the rural areas apparently retreated from its spring counterpart as a result of rice transplanting.

\subsection{The Refinement of Urban Land Cover Components}

Due to the confusion between soil and HA impervious surface and between water and LA impervious surface, the conventional method of directly adding HA and LA fractions together to estimate impervious surfaces may not work here. The refinement procedure devised a postprocessing mechanism to separate the targeted land cover type (e.g., imperviousness in this case) from the preliminary fractions by utilizing the LST and the coarse component fractions derived from the two-season images.

The first task here is to eliminate perennial water bodies from LA fractions through an LA fraction-to-LST ratio based filter. As discussed before, pixels with a high LA fraction-to-LST ratio in both seasons can be assumed to be perennial water, since lakes and ponds are always characterized with a high LA fraction and a low LST value, as evident in Fig. 3. The LA fractionto-LST ratio maps for the two test sites are shown in Fig. 5. It should be pointed out that urban shadows existent in the spring map due to their relatively high LA fraction-to-LST ratio [Fig. 5(a)] were suppressed in summer [Fig. 5(b)], ensuring their distinction from perennial water. The perennial water bodies were separated by selecting an LA fraction-to-LST ratio value of 0.003 as the threshold for both seasons with the help of visual interpretation of aerial imagery.

The second task involved separating seasonal water bodies from LA via change detection analysis. Rice paddies and temporary fish farming ponds are the major cause of the LA fraction increase from spring to summer. Comparing the summer LA fraction to its spring counterpart offered an easy removal of water-induced LA fraction. Similarly to the threshold determination 
(a)

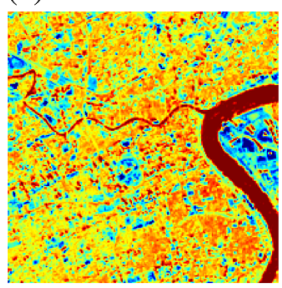

(b)

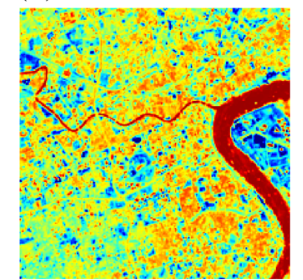

(c)

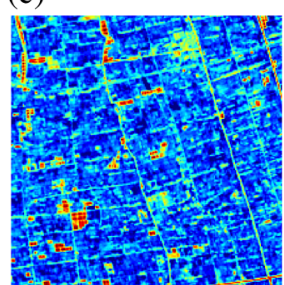

(d)

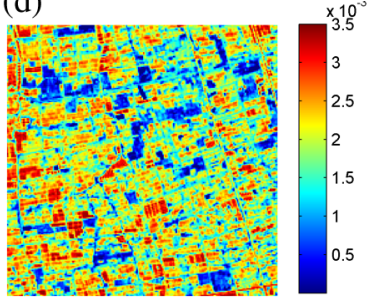

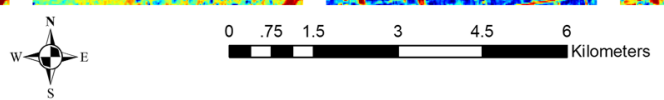

Fig. 5 The LA fraction-to-LST ratio maps of two selected test sites in different seasons [(a) urban area in spring; (b) urban area in summer; (c) rural area in spring; and (d) rural area in summer].

for the LA fraction-to-LST ratio, pixels with a between-season LA fraction difference above 0.3 was considered seasonal water, whereas those below this threshold were deemed to be impervious surfaces.

In the third task, the spring soil pixels covered by summer vegetation were subtracted from the preliminary HA fraction so that the true HA imperviousness could hopefully emerge. HA fraction decreased significantly from spring to summer in the rural areas due to much exposed soil being later covered by full-blown vegetation [Fig. 4(a)]. As a result of empirical thresholding, therefore, a pixel was reclassified as soil if it simultaneously met the following two criteria: the amount of HA reduction from spring to summer was greater than 0.2 , and the summer HA fraction was less than 0.1. The HA soil fraction was then subtracted from the preliminary HA fraction, leaving the rest as the HA impervious surface.

The above refining process generated five land cover fraction maps for each season (Fig. 6), including LA impervious surface, water (perennial and seasonal water), HA impervious surface, soil, and vegetation. The "rainbow" color ramp was used to represent the fraction variation, with red for unit fraction (1) and blue for zero fraction (0). After removing water features, the remaining LA fractions were considered a good representation of LA impervious surfaces, typically showing up in urbanized areas and district town seats [Fig. 6(a)]. In Shanghai, old building roofs and the ground paved with asphalt were usually the major contributors to the high level of LA imperviousness. On the other hand, the HA impervious surfaces were dominant in the regions around the outer loop and some new cities [Fig. 6(c)], where the new urban constructions took place. Both LA and HA impervious surfaces appeared highly similar in both seasons, with just a slight difference due to vegetation phenology for both urban or rural sites. In contrast, the seasonal change of water distribution patterns varied drastically between the urban and rural sites.

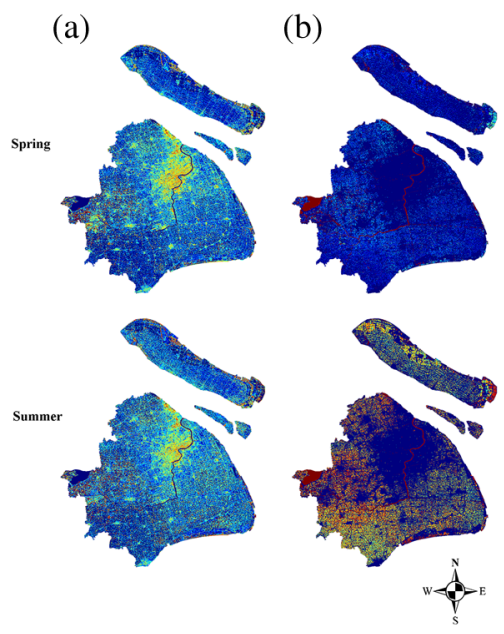

(c)

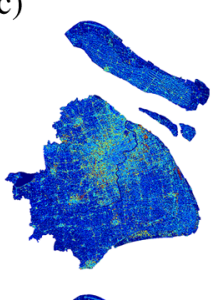

(d)
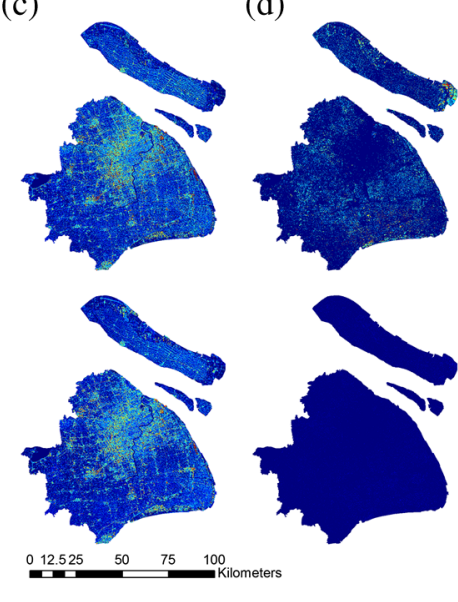

(e)

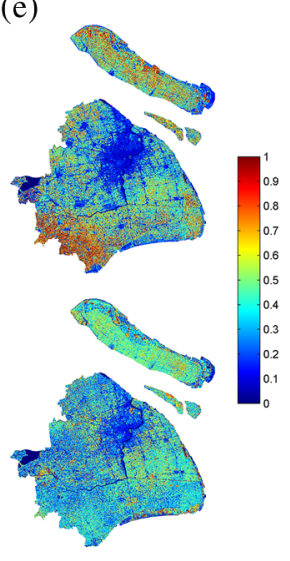

Fig. 6 Fraction maps of five land cover types [(a) LA impervious surface; (b) water; (c) HA impervious surface; (d) soil, and (e) vegetation]. The first row denotes the spring results and the second row represents the summer situation. 
Wang, Yao, and Ji: Integrating seasonal optical and thermal infrared spectra to characterize urban...

Urban water bodies are mostly perennial, so little change can be seen from season to season, whereas seasonal water features are mostly located in the rural area in summer, leading to a significant increase in water fractions from spring to summer [Fig. 6(b)]. Spring soils were found scattered in the rural area, while bare soil became minimal in summer [Fig. 6(d)]. This is due to the regrowth of vegetation in the urban area and mainly owing to crop rotation in the rural area. Vegetation fraction maps [Fig. 6(e)] remained the same as the coarse land cover decomposition results.

\subsection{Evaluation of Estimated Impervious Surfaces}

The end products of impervious surfaces were generated by adding together the HA and LA impervious surface fraction images for each season. ${ }^{3}$ These products are shown in Fig. 7, along with their original ETM+ images. Overall, the spatial pattern of the impervious surface fractions seemed to agree well with the visual features on the original images. The imperviousness concentrated in the urban center areas inside the outer loop, district seats scattered in the rural areas, and the road network connecting the metropolitan area and district seats. On the other hand, the distribution patterns of impervious surfaces were relatively consistent between the two seasons, except that this feature seemed much more intensified in spring than in summer,

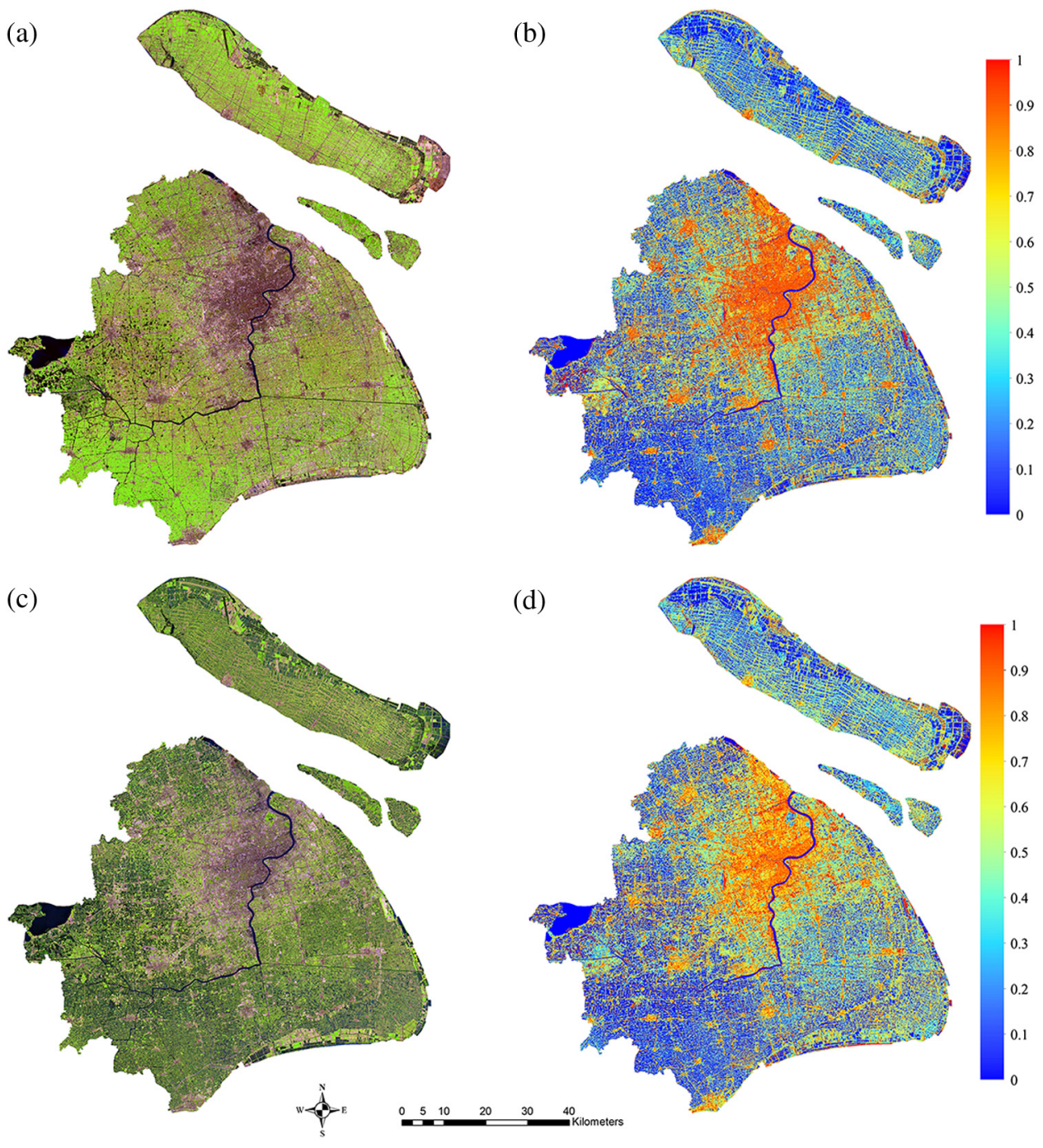

Fig. 7 Fraction maps of impervious surface on (b) March 13, 2001, and (d) July 3, 2001, and the original ETM+ images with the color composite RGB $=543$ on (a) March 13, 2001, and (c) July 3, 2001. 
Wang, Yao, and Ji: Integrating seasonal optical and thermal infrared spectra to characterize urban...

Table 1 Accuracy measures of different methods used in urban and rural areas.

\begin{tabular}{|c|c|c|c|c|c|}
\hline \multirow[b]{2}{*}{ Metric } & \multirow[b]{2}{*}{ Test Site } & \multicolumn{2}{|c|}{ Two-step decomposition } & \multicolumn{2}{|c|}{ Traditional method } \\
\hline & & Spring & Summer & Spring & Summer \\
\hline \multirow[t]{3}{*}{ RMSE } & Urban area & 0.147 & 0.137 & 0.249 & 0.162 \\
\hline & Rural area & 0.179 & 0.147 & 0.267 & 0.246 \\
\hline & Overall & 0.166 & 0.140 & 0.242 & 0.206 \\
\hline \multirow[t]{3}{*}{ MAE } & Urban area & 0.119 & 0.116 & 0.215 & 0.129 \\
\hline & Rural area & 0.140 & 0.116 & 0.230 & 0.202 \\
\hline & Overall & 0.129 & 0.116 & 0.206 & 0.168 \\
\hline
\end{tabular}

especially in the urbanized areas. This difference could be attributed to the low canopy closure of urban vegetation in spring and the full-blown canopy closure in summer, as shown in the original ETM+ images [Fig. 7(a) and 7(c)]. The significant increase of pixels mixing vegetation and imperviousness in the summer image seemed to be the major cause for the degradation of imperviousness detection.

In addition to visual comparisons, quantitative accuracy assessment was performed to evaluate the estimated results of impervious surfaces derived using the proposed approach. For the purpose of cross-method comparison, imperviousness fractions directly derived using a four-endmember LSMA model (HA, LA, soil, and vegetation) with a single image ${ }^{3}$ (termed "traditional method" hereafter) were also evaluated. Three quality indicators, RMSE, MAE, and $R^{2}$, were calculated for the impervious surfaces fraction images for the two seasons at the typical urban and rural test sites. The results are summarized in Table 1 and Fig. 8.

Several important observations can be made from the assessment results. First, the overall accuracy of the imperviousness fractions derived using the proposed approach is rather high, as both RMSE and MAE are below 0.20 and $R^{2}$ values are greater than 0.70 for all test sites for both seasons, indicating that the two-step decomposition strategy was effective and reliable. Compared with the proposed method, however, the estimates made by the traditional method were much less accurate. The overall RMSE and MAE for the traditional method were significantly higher than those for the two-step method (ranging from $45 \%$ to 59\%), and $R^{2}$ was significantly lower (ranging from $19.8 \%$ to $37.2 \%$ ). In terms of scatter plot analysis, the summer
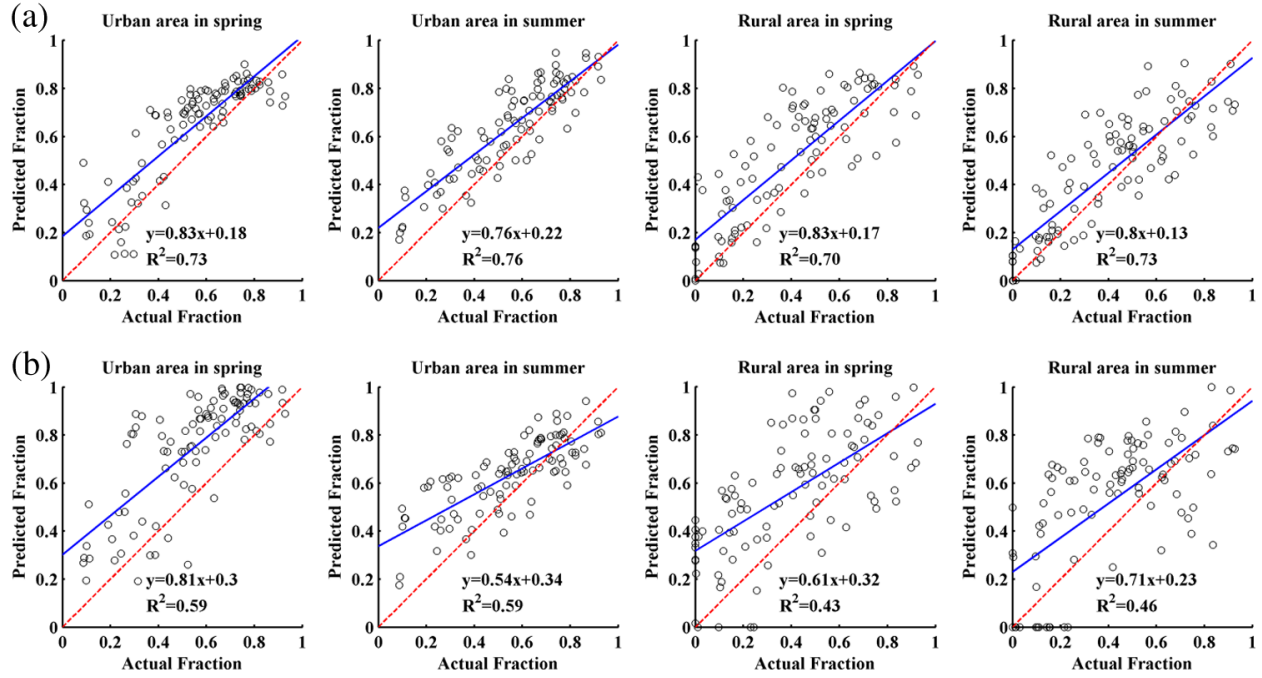

Fig. 8 Scatter plots of the actual fraction versus predicted fraction derived from the spring and summer images by the (a) new method and (b) traditional method in the urban and rural area (the dashed line is the $1: 1$ reference line, and the solid line is the regression line). 
Wang, Yao, and Ji: Integrating seasonal optical and thermal infrared spectra to characterize urban...

map seems more accurate than the spring map. For both the proposed and traditional methods, the statistical accuracy of the summer fractions was higher than that of spring, indicating that the summer image was a better choice for impervious surfaces estimation. Second, urban areas present lower RMSE and MAE values than the rural test sites regardless of season.

\section{Discussion}

When remote sensing is used to map urban imperviousness, spectral resemblance among different land cover types is the first issue to be considered. ${ }^{1}$ The major confused cover types include water and soil. The very essence of the two-step decomposition design is to introduce multitemporal optical and thermal data to improve both the precision and accuracy of impervious surface coverage by eliminating spectral confusion from water and soils. The relatively high RMSE and MAE and low $R^{2}$ values for the results based on the traditional method might be caused by the following problems: the misclassification of bare soils as bright impervious surfaces and the confusion between water and LA imperious surfaces. These are exactly the problems that have been properly handled by the proposed method in this study (Fig. 8 and Table 1).

The improved results of this study further confirmed the findings of other scholars who also utilized temporal change detection analyses for estimating land covers. ${ }^{19,26-28}$ In comparison with these studies, we focused on change detection using the fraction images developed from LSMA, which can provide more accurate land cover features than pixel-based original spectral, ${ }^{28}$ NDVI or other vegetation indices. ${ }^{26,27}$ The only exception is the study of Powell et al., ${ }^{19}$ where they used time-series fraction images to identify impervious surfaces, but their large temporal interval (years) was inadequate for rapid-growth cities like Shanghai. Change detection based on temporal fractions in the two-step decomposition procedure was similar to the conventional fractions change detection analysis but served a different purpose, i.e., to refine extraction of certain land cover that has significant spectral confusion with other land cover types. ${ }^{29}$

It should also be noted that the two-step decomposition analysis was performed on images acquired within a one-year period (2001/03/13 and 2001/07/03), ensuring that the true change of impervious surfaces fractions was kept minimal. However, seasonal effects on impervious surface estimation were still observed, and the better performance was achieved with the summer image, which was similar to the conclusion reported in other studies. ${ }^{1,30,31}$ The reason for this is because LSMA partitions data variance by a principal component transformation of multispectral imagery and mixing space characterization. ${ }^{32}$ The summer image may optimize the mixing space (HA, LA, and vegetation) with the full growth of vegetation, thus leading to a more accurate estimation of impervious surfaces. On the other hand, the function of the spring image in the proposed method is to provide seasonal cues for eliminating water, soil, and shadow pixels from the HA and LA induced from the summer image. Therefore, pairing images of two seasons in the analysis to detect imperviousness by best utilizing information from plant phenology and local farming practices is a feasible and effective strategy to detailed and accurate land cover classification.

The potential applications of LST to identify water from LA were demonstrated in many previous studies. ${ }^{1,13}$ However, a single LST threshold is insufficient to fully delineate water bodies because they are not unique in thermal properties among other urban features. In this study, the ratio of LA fraction and LST was proved more adequate for removal of water from the LA fraction image, thus achieving a more reliable impervious surfaces estimation (Table 1 and Fig. 8). It is worth noting that the spectral and thermal characteristics of some shadows in urban areas are nearly identical to water bodies (Fig. 3), but the two seasonal LA fraction-to-LST ratio maps are capable of distinguishing shadow from water because of the season-to-season change of shadow length and orientation. As can be observed in the water fraction maps in urban areas (Fig. 6), shadows cast by tall buildings in CBD were not misclassified as water bodies. The shadow problem in land cover classification has been pending for a long time, especially for high-resolution satellite images. ${ }^{33}$ The successful application of temporal LA fraction-to-LST ratio in this study may shed some light on future shadow detection. 


\section{Conclusion}

This research focused on improving urban impervious surface mapping by developing a two-step land cover decomposition method. The method exploited optical and thermal data from two seasons to eliminate water from LA impervious surface and soil from HA impervious surface. This approach was tested in Shanghai using two Landsat ETM+ images acquired in spring and summer of 2001, respectively. Validated by reference data derived from aerial photographs, the test results showed a noticeable improvement in impervious surface estimation with the two-step decomposition method. Although only validated with data from Shanghai in this case study, the approach is not location specific and can be applied elsewhere. The development of new satellite remote sensing missions has led to an increasing amount of multitemporal data, and the fusion of multisource remote sensing data has been demonstrated as a promising approach to provide more useful information. Future research should focus on the combinations of multitemporal and multisource data for land cover sub pixel decomposition, especially in the highly heterogeneous and complex urban environments.

\section{Acknowledgments}

We would like to acknowledge the anonymous reviewers for their constructive and valuable suggestions on the earlier drafts of this manuscript. This research was supported by National Natural Science Foundation of China (Grant No. 41271055), Natural Science Foundation of Shanghai (Grant No. 15ZR1436800), Outstanding Doctoral Dissertation Cultivation Plan of Action (Grant No. PY2014013), and China Scholarship Council (Grant No. 201406140057).

\section{References}

1. Q. Weng, X. Hu, and H. Liu, "Estimating impervious surfaces using linear spectral mixture analysis with multitemporal ASTER images," Int. J. Remote Sens. 30(18), 4807-4830 (2009).

2. F. Yuan et al., "Land cover classification and change analysis of the Twin Cities (Minnesota) Metropolitan Area by multitemporal Landsat remote sensing," Remote Sens. Environ. 98(2), 317-328 (2005).

3. C. Wu and A. T. Murray, "Estimating impervious surface distribution by spectral mixture analysis," Remote Sens. Environ. 84(4), 493-505 (2003).

4. D. Lu, E. Moran, and S. Hetrick, "Detection of impervious surface change with multitemporal Landsat images in an urban-rural frontier," ISPRS J. Photogramm. Remote Sens. 66(3), 298-306 (2011).

5. F. Fan and W. Fan, "Understanding spatial-temporal urban expansion pattern (1990-2009) using impervious surface data and landscape indexes: a case study in Guangzhou (China)," J. Appl. Remote Sens. 8(1), 083609 (2014).

6. C. $\mathrm{Wu}$, "Normalized spectral mixture analysis for monitoring urban composition using ETM+ imagery," Remote Sens. Environ. 93(4), 480-492 (2004).

7. H. Xu, "Analysis of impervious surface and its impact on urban heat environment using the normalized difference impervious surface index (NDISI)," Photogramm. Eng. Remote Sens. 76(5), 557-565 (2010).

8. L. Ji, L. Zhang, and B. Wylie, "Analysis of dynamic thresholds for the normalized difference water index," Photogramm. Eng. Remote Sens. 75(11), 1307-1317 (2009).

9. H. Xie et al., "New hyperspectral difference water index for the extraction of urban water bodies by the use of airborne hyperspectral images," J. Appl. Remote Sens. 8(1), 085098 (2014).

10. B. C. Gao, "NDWI-A normalized difference water index for remote sensing of vegetation liquid water from space," Remote Sens. Environ. 58(3), 257-266 (1996).

11. S. McFeeters, "The use of the normalized difference water index (NDWI) in the delineation of open water features," Int. J. Remote Sens. 17(7), 1425-1432 (1996).

12. H. Xu, "Modification of normalised difference water index (NDWI) to enhance open water features in remotely sensed imagery," Int. J. Remote Sens. 27(14), 3025-3033 (2006). 
Wang, Yao, and Ji: Integrating seasonal optical and thermal infrared spectra to characterize urban...

13. D. Lu and Q. Weng, "Use of impervious surface in urban land-use classification," Remote Sens. Environ. 102(1-2), 146-160 (2006).

14. A. R. As-Syakur et al., "Enhanced built-up and bareness index (EBBI) for mapping built-up and bare land in an urban area," Remote Sens. 4(10), 2957-2970 (2012).

15. H. Xu, D. Lin, and F. Tang, "The impact of impervious surface development on land surface temperature in a subtropical city: Xiamen, China," Int. J. Climatol. 33(8), 1873-1883 (2013).

16. C. Deng and C. Wu, "Examining the impacts of urban biophysical compositions on surface urban heat island: a spectral unmixing and thermal mixing approach," Remote Sens. Environ. 131(4), 262-274 (2013).

17. H. Zhao and C. Xiaoling, "Use of normalized difference bareness index in quickly mapping bare areas from TM/ETM+," in Proc. 2005 IEEE Int. on Geoscience and Remote Sensing Symp. (IGARSS '05), pp. 1666-1668 (2005).

18. M. K. Ridd, "Exploring a VIS (vegetation-impervious surface-soil) model for urban ecosystem analysis through remote sensing: comparative anatomy for cities," Int. J. Remote Sens. 16(12), 2165-2185 (1995).

19. S. L. Powell et al., "Quantification of impervious surface in the Snohomish water resources inventory area of western Washington from 1972-2006," Remote Sens. Environ. 112(4), 1895-1908 (2008).

20. P. T. Wolter et al., "Improved forest classification in the Northern Lake States using multi-temporal Landsat imagery," Photogramm. Eng. Remote Sens. 61(9), 1129-1144 (1995).

21. M. Ji and J. R. Jensen, "Effectiveness of subpixel analysis in detecting and quantifying urban imperviousness from Landsat thematic mapper imagery," Geocarto Int. 14(4), 33-41 (1999).

22. Y. Zhang, "Detection of urban housing development by fusing multisensor satellite data and performing spatial feature post-classification," Int. J. Remote Sens. 22(17), 3339-3355 (2001).

23. X. Dai et al., "Spatio-temporal pattern of urban land cover evolvement with urban renewal and expansion in Shanghai based on mixed-pixel classification for remote sensing imagery," Int. J. Remote Sens. 31(23), 6095-6114 (2010).

24. United States Geological Survey, “Global visualization viewer," http://www.usgs.gov/ (19 April 2013).

25. J. A. Sobrino, J. C. Jiménez-Muñoz, and L. Paolini, "Land surface temperature retrieval from LANDSAT TM 5," Remote Sens. Environ. 90(4), 434-440 (2004).

26. P. Sinha and L. Kumar, "Independent two-step thresholding of binary images in inter-annual land cover change/no-change identification," ISPRS J. Photogramm. Remote Sens. 81(7), 31-43 (2013).

27. W. Kleynhans et al., "Improving land cover class separation using an extended Kalman filter on MODIS NDVI time-series data," IEEE Geosci. Remote Sens. Lett. 7(2), 381-385 (2010).

28. M. S. Gilmore et al., "Integrating multi-temporal spectral and structural information to map wetland vegetation in a lower Connecticut River tidal marsh," Remote Sens. Environ. 112(11), 4048-4060 (2008).

29. D. Lu, M. Batistella, and E. Moran, "Multitemporal spectral mixture analysis for Amazonian land-cover change detection," Can. J. Remote Sens. 30(1), 87-100 (2004).

30. C. Wu and F. Yuan, "Seasonal sensitivity analysis of impervious surface estimation with satellite imagery," Photogramm. Eng. Remote Sens. 73(12), 1393-1401 (2007).

31. X. Hu and Q. Weng, "Estimation of impervious surfaces of Beijing, China, with spectral normalized images using linear spectral mixture analysis and artificial neural network," Geocarto Int. 25(3), 231-253 (2010).

32. C. Small and J. W. T. Lu, "Estimation and vicarious validation of urban vegetation abundance by spectral mixture analysis," Remote Sens. Environ. 100(4), 441-456 (2006).

33. P. M. Dare, "Shadow analysis in high-resolution satellite imagery of urban areas," Photogramm. Eng. Remote Sens. 71(2), 169-177 (2005).

Wei Wang is currently a PhD student in geographic information science at East China Normal University. She holds an MS degree in agronomy from Nanjing Agricultural University. Her 
Wang, Yao, and Ji: Integrating seasonal optical and thermal infrared spectra to characterize urban...

research areas include remote sensing of environment, remote sensing of nutrient content of crop leaves, and urban dynamic modeling with geospatial technology.

Xinfeng Yao received his PhD in agronomy from Nanjing Agricultural University in 2012. Currently, he is an assistant researcher at the Agricultural Information Institute of Science and Technology, Shanghai Academy of Agricultural Sciences. His major research interests include agricultural information technology and remote sensing of vegetation.

Minhe Ji is currently a professor of geography at East China Normal University. He received his $\mathrm{PhD}$ from the University of South Carolina in 1996 and his MS degree from Northern Illinois University in 1990. Before joining the faculty at ECNU, he held a professorship at the University of North Texas. His research interests include digital image analysis, fuzzy set theory for geospatial data processing, spatial statistics, and AI applications in transport information service. 